Liver, Pancreas and Biliary Tract

\title{
Autoimmunity and lymphoproliferation markers in naïve HCV-RNA positive patients without clinical evidences of autoimmune/lymphoproliferative disorders
}

\author{
Francesca Gulli ${ }^{a, 1}$, Umberto Basile ${ }^{a, 1}$, Laura Gragnani ${ }^{\mathrm{b}, *, 1}$, Elisa Fognani ${ }^{\mathrm{b}}$, \\ Cecilia Napodano ${ }^{\mathrm{a}}$, Luigi Colacicco ${ }^{\mathrm{a}}$, Luca Miele $^{\mathrm{c}}$, Nicoletta De Matthaeis ${ }^{\mathrm{c}}$, Paola Cattani ${ }^{\mathrm{d}}$, \\ Anna Linda Zignego ${ }^{\mathrm{b}}$, Gian Ludovico Rapaccini ${ }^{\mathrm{c}}$ \\ a Department of Laboratory Medicine of the Catholic University of the Sacred Heart, Rome, Italy \\ b Center for Systemic Manifestations of Hepatitis Viruses (MaSVE), Department of Experimental and Clinical Medicine, University of Florence, Florence, Italy \\ ${ }^{c}$ Institute of Internal Medicine, Catholic University of the Sacred Heart, Rome, Italy \\ ${ }^{\mathrm{d}}$ Institute of Microbiology, Catholic University of the Sacred Heart, Rome, Italy
}

\section{A R T I C L E I N F O}

\section{Article history:}

Received 12 January 2016

Accepted 17 May 2016

Available online 24 May 2016

\section{Keywords:}

Antinuclear antibodies (ANA)

Cryoglobulins (CGs)

Hepatitis C virus (HCV)

Rheumatoid factor (RF)

\begin{abstract}
A B S T R A C T
Background: HCV can lead to both chronic liver disease and B-cell lymphoproliferative disorders. A strong association exists between HCV and mixed cryoglobulinaemia (MC).

Methods: Anti-nuclear antibodies (ANA), rheumatoid factor Ig-G (RF-IgG), free light chain $\kappa$ and $\lambda$ (FLC-к, FLC- $\lambda$ ) levels and $\kappa / \lambda$ ratio were evaluated in $50 / 420$ subjects unexpectedly resulted anti-HCV positive after routine screenings for non-hepathological procedures.

Results: Three/fifty patients had HCV-RNA undetectable in the serum and were excluded from the analysis. Thirty-nine/fifty patients had laboratory evidence of circulating cryoglobulins without liver disease and MC-related symptoms. Among them, 17 resulted ANA-positive.

The mean cryocrit was higher in ANA-positive patients, while no other demographic/clinical differences were observed between the groups. Significantly higher levels of RF-IgG were observed in ANA-positive vs ANA-negative patients. $\kappa$ and $\lambda$ FLC were higher in ANA-positive patients.

A ROC analysis, based on ANA-positivity vs ANA-negativity, confirmed a high sensitivity and specificity of RF-IgG test.

Conclusions: Published data concerning MC come mostly from symptomatic vasculitis. We analyzed HCVpatients without MC symptoms, founding cryoglobulins in the majority of them.

The increased levels of FR-IgG and FLC in CGs-ANA-positive patients, suggest these test could be used to identify a state of silent autoimmune and/or lymphoproliferative condition before the transition to a frank disease in naïve HCV-patients without symptoms of extrahepatic manifestations.
\end{abstract}

(c) 2016 Editrice Gastroenterologica Italiana S.r.l. Published by Elsevier Ltd. All rights reserved.

\section{Introduction}

Hepatitis C virus (HCV) infects about 200 million people world-wide, leading to both chronic liver disease and B-cell lymphoproliferative disorders (LPDs). A strong association has been

\footnotetext{
Corresponding author at: Center for Systemic Manifestations of Hepatitis Viruses (MASVE), Department of Experimental and Clinical Medicine, University of Florence, Largo Brambilla 3, 50134 Florence, Italy. Tel.: +39 055 2758087; fax: +39055 2758099.

E-mail address: laura.gragnani@unifi.it (L. Gragnani).

1 Contributed equally to this work.
}

shown between HCV and mixed cryoglobulinaemia (MC), a benign, but pre-lymphomatous condition $[1,2]$.

The activation of B-lymphocytes is in turn responsible for the production of immune complexes, including cryoglobulins (CGs) and various autoantibodies [2,3]. CGs are immunoglobulins which undergo reversible precipitation or gelling when exposed to temperatures below $37^{\circ} \mathrm{C}$ and re-dissolve upon rewarming [4]. Their cold-insolubility is still unclear and may depend upon variety of factors. Low temperatures seem to trigger a reversible cryoprecipitation, possibly by inducing steric modifications of the Ig molecules [4]. Immunochemical characterization of CGs has led to their classification into three major groups according to Brouet's classification [5]. 
Type I represents individual monoclonal immunoglobulins, generally associated with lymphoproliferative diseases; type II consists of mixed immunoglobulins with a monoclonal/oligoclonal IgM component and is strongly associated with HCV infection; type III is constituted by a mixture of polyclonal IgMs and IgGs and is associated with a wide range of infectious (including HCV), autoimmune and liver diseases $[4,6]$. Types II and III are referred as MC, which implies the presence of circulating immune complexes composed of polyclonal IgGs (behaving as auto-antigens) and IgMs (behaving as auto-antibodies), with rheumatoid factor (RF) activity.

HCV infection may trigger a collection of immunological mechanisms responsible for the development of different autoimmune diseases $[2,3]$. Anti-nuclear antibodies were recently detected in cryoprecipitates from HCV-positive sera [7] and some patients with chronic HCV infection are anti-liver and kidney microsomal antibody type 1 (anti-LKM1) positive with the autoantibody titre correlating with disease severity [8]. Also, the presence of autoantibodies, RF-IgG and IgG3 in the cryoprecipitates from HCVpositive patients suggests a strong activation of the immune system [7].

Cryofibrinogenemia refers to the presence, in serum, of a cryoprotein that was first identified in 1955 by Korst and Kratochvil. Unlike cryoglobulinaemia, the precipitate forms only in plasma and not in the serum [9].

Serum free light chains (FLCs), $\kappa$ and $\lambda$, have been identified as useful markers whose alteration is linked to specific diseases; the quantitative assay of $K$ chains, $\lambda$ chains and $\kappa / \lambda$ ratio is a diagnostic tool in plasma cell dyscrasias, such as multiple myeloma, monoclonal gammopathy of undetermined significance and amyloidosis [10-12]. An abnormal $\kappa / \lambda$ FLC ratio is interpreted as a surrogate for clonal expansion [13].

The fast turnover of FLC, particularly true for the $\kappa$ chain (less than $6 \mathrm{~h}$ compared to 20-25 days to total $\operatorname{IgG}$ ), explains that they are considered ideal markers of response in patients undergoing rituximab treatment [14].

The aim of this study was therefore to find a panel of analytic tests, easy to assess, that could identify a state of silent autoimmune and/or lymphoproliferative condition before the transition to a frank disease in naïve HCV patients without symptoms of extrahepatic manifestations.

\section{Materials and methods}

\subsection{Patients}

Naïve patients with HCV infection were consecutively recruited from January 2014 to December 2014 by the Department of Gastroenterology of the Institute of Internal Medicine, Hospital Foundation "A. Gemelli", Catholic University of Sacred Hearth, Rome Italy. All the enrolled subjects resulted HCV-RNA positive after routine screenings performed before undergoing different non-hepatological procedures. Subjects were included in the study according to the following criteria: no previous antiviral treatments, absence of symptoms of hepatic disease and autoimmune and/or LPDs and normal levels of C4 and C3. Exclusion criteria were: presence of abnormal ALT values, renal involvement or other symptoms possibly related to $\mathrm{MC}$ vasculitis, inflammatory diseases with elevated levels of C-reactive protein (CRP) or co-infections (HIV and HBV). All the patients signed a written informed consent, in accordance with the Principles of the Declaration of Helsinki.

\subsection{Laboratory findings}

The quantitative HCV-RNA detection was assessed by routine method and virus genotype was determined for each sample
(Siemens Healthcare, Germany). Briefly, presence and quantification of HCV-RNA were determined by means of real-time polymerase chain reaction (PCR), transcription-mediated amplification (TMA), and multi-probe reverse hybridization of the 5_untranslated region (5_UTR) of the HCV genome. Results were reported in $\mathrm{IU} / \mathrm{mL}$. Testing for $\mathrm{HCV}$ genotype and subtype was performed through reverse transcription and PCR (RT-PCR) and reverse hybridization of the HCV genome. Genotypes and subtypes detectable through this test include $1 \mathrm{a}, 1 \mathrm{~b}, 2 \mathrm{a} / 2 \mathrm{c}, 2 \mathrm{~b}, 3 \mathrm{a}, 3 \mathrm{~b}, 3 \mathrm{c}, 4 \mathrm{a}$, $4 \mathrm{~b}, 4 \mathrm{c} / 4 \mathrm{~d}, 4 \mathrm{e}, 4 \mathrm{f}, 4 \mathrm{~h}, 5 \mathrm{a}$ and $6 \mathrm{a}$.

For CGs detection, $10 \mathrm{~mL}$ of peripheral blood were collected and immediately stored at $37{ }^{\circ} \mathrm{C}$ in pre-warmed tubes without anticoagulant for $30 \mathrm{~min}$ to enable complete blood clotting. Serum was transferred in Wintrobe tubes and stored for at least 7 days at $4{ }^{\circ} \mathrm{C}$ in order to evaluate the presence of precipitates and flocculation. Before immunologic testing, the cryocrit percentage was assessed, the supernatant was removed and stored for subsequent analyses. The remaining cryoprecipitate was recovered and washed 3 times. Cryoprecipitate was re-suspended with an appropriate volume of 3\% PEG 6000 solution and re-solubilized for $30 \mathrm{~min}$ at $37^{\circ} \mathrm{C}$. CGs were characterized by immunofixation electrophoresis (IFE) with G26 Fully automated system (Interlab, Italy) [15]. To detect cryofibrinogenemia a similar analytical procedure has been used but peripheral blood was collected in tubes containing anticoagulants (oxalate, citrate, or ethylenediamine tetraacetic acid). Heparin-coated tubes should not be used to avoid false-positive results due to the formation of a cryoprecipitate containing fibronectin, fibrin and fibrinogen together with heparin, known as "heparin-precipitable fraction" [16]. Antinuclear antibody (ANA) determination was carried out by indirect immuno-fluorescence (IIFA) [17]. IIFA entails substantial technical expertise and it is considered real gold standard assay. Briefly, all serum specimens were titrated in 1:160 in order to avoid false-positive non-specific low titre dilution. Diluted serum was added to HepG2 cells (ATCC, IL-INOVA, USA) at $37^{\circ} \mathrm{C}$. A secondary polyclonal antibody (anti-human IgG FITC-conjugated polyclonal sheep antiserum) was subsequently added. Slides were fixed with glycerol and the staining pattern evaluated by microscopy [18].

Liver-dot, an immunodot blot kit (AXA diagnostics Srl, Italy), has been used to detect, in the serum, IgG-autoantibodies directed against the following antigens: M2/nPDC, M2/OGDC-E2, M2/BCOADC-E2, M2/PDC-E2, gp210, sp100, LKM1, LC1 and SLA [19]. Samples were tested at $37^{\circ} \mathrm{C}$ according to the manufacturer's instructions and serum dilutions, where necessary, were performed following the recommendations.

FLCs were assessed by means of Turbidimetric assay (Freelite TM Human Kappa and Lambda Free Kits, The Binding Site, UK) and performed on the SPAplus instrument (The Binding Site, UK). Samples were tested at $37^{\circ} \mathrm{C}$ and serum dilutions were performed if needed.

Rheumatoid factor-IgG (RF-IgG) was tested using ELISA kits for RF-IgG (INOVA-diagnostics, USA) and performed on the ELISA and IIFA SkyLAB 752 analyzer automatic system (DASIT-Italy).

ROC curves (Receiver Operating Characteristic) were generated by plotting data on GraphPad Prism (GraphPad Software, Inc., S. Diego, CA, USA) and the resulting information was then re-plotted on Microsoft Excel (Microsoft Corporation, Redmond, WA, USA). Data from FLC and RF-IgG serum concentrations were plotted against ANA positivity/negativity, in order to give estimates of true positive values (sensitivity) and the proportion of false negatives (specificity). Plotted values are represented as a curve, and the Area Under the Curve (AUC) indicates diagnostic accuracy. An AUC $=1$ (100\%) denotes full accuracy of the test. In this way, it is possible to identify the best cut-off value for a specific test and to discriminate normal from abnormal values. 
Table 1

Main baseline characteristics of the $39 \mathrm{HCV}$ patients with circulating cryoglobulins.

\begin{tabular}{|c|c|c|c|c|}
\hline & $\begin{array}{l}\text { All patients } \\
(n=39)\end{array}$ & $\begin{array}{l}\text { ANA positive } \\
(n=17)\end{array}$ & $\begin{array}{l}\text { ANA negative } \\
(n=22)\end{array}$ & $p$ value \\
\hline Mean age (years) & $61.74 \pm 1.48$ & $60.65 \pm 2.13$ & $62.59 \pm 2.07$ & ns \\
\hline Sex (male/female) & $15 / 24$ & $4 / 13$ & $11 / 11$ & ns \\
\hline \multicolumn{5}{|l|}{ HCV genotype: } \\
\hline $1(\%)$ & 36 & 16 & 20 & \\
\hline $2(\%)$ & 2 & - & 2 & \\
\hline $3(\%)$ & 1 & 1 & - & \\
\hline $4(\%)$ & - & - & - & \\
\hline Viral titre $\left(\mathrm{IU} / \mathrm{mL} \times 10^{5}\right)$ & $7.46 \pm 2.66$ & $5.61 \pm 2.11$ & $8.90 \pm 4.45$ & ns \\
\hline Mean ALT $(\mathrm{U} / \mathrm{L})^{\mathrm{a}}$ & $32.49 \pm 1.07$ & $35.01 \pm 2.44$ & $30.50 \pm 2.32$ & ns \\
\hline \multicolumn{5}{|l|}{ Characteristics of MC: } \\
\hline Cryocrit \% & $2.6 \pm 0.2$ & $3.3 \pm 0.4$ & $2.2 \pm 0.3$ & 0.026 \\
\hline Type II & $18 / 39(46.2 \%)$ & $10 / 17(58.8 \%)$ & $8 / 22(36.4 \%)$ & ns \\
\hline Type III & $21 / 39(53.8 \%)$ & $7 / 17(41.2 \%)$ & $14 / 22(63.6 \%)$ & \\
\hline RF alteration & $8 / 39(20.51 \%)$ & $2 / 17(11.8 \%)$ & $6 / 22(27.2 \%)$ & ns \\
\hline \multicolumn{5}{|l|}{ Serum FLC alteration: } \\
\hline$\kappa$ & $34 / 39(87.2 \%)$ & $17 / 17(100 \%)$ & $17 / 22(77.2 \%)$ & ns \\
\hline$\lambda$ & $21 / 39(53.4 \%)$ & $11 / 17(64.7 \%)$ & $10 / 22(45.5 \%)$ & ns \\
\hline FLC ratio & $13 / 39(33.3 \%)$ & $9 / 17(52.9 \%)$ & $4 / 22(18.2 \%)$ & 0.0392 \\
\hline
\end{tabular}

Values are expressed as mean \pm standard error mean (SEM).

ns, not significant; IU, international units; MC, mixed cryoglobulinemia; RF, rheumatoid factor; FLC, free light chains.

a Normal range for ALT: 7-45 U/L.

\subsection{Statistical analysis}

Collected data sets were reported on Microsoft Excel TM worksheets (Microsoft, Redmond, WA, USA) and was computed using Statgraphics Plus 5.1 (STATPOINT TECHNOLOGIES, INC., Warrenton, Virginia, USA) e Graph-Pad Prism (GraphPad Software, Inc., S. Diego, CA, USA). Data are expressed as mean \pm standard error mean (SEM). The Chi-square and Fisher tests were used to evaluate categorical variables. Statistical significance was set at a value of $p<0.05$.

\section{Results}

From January 2014 to December 2014, 420 patients were examined in the Department of Gastroenterology of the Institute

A Type II and III cryo-patients

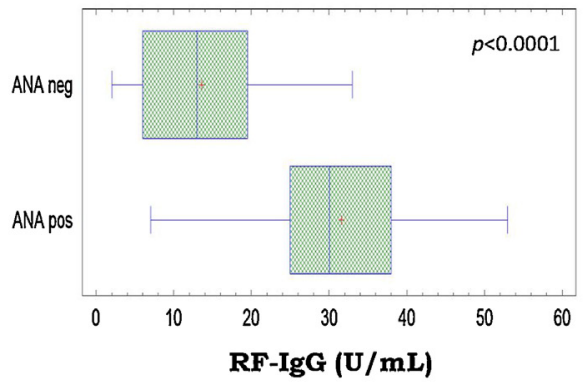

C Type II and III cryo-patients

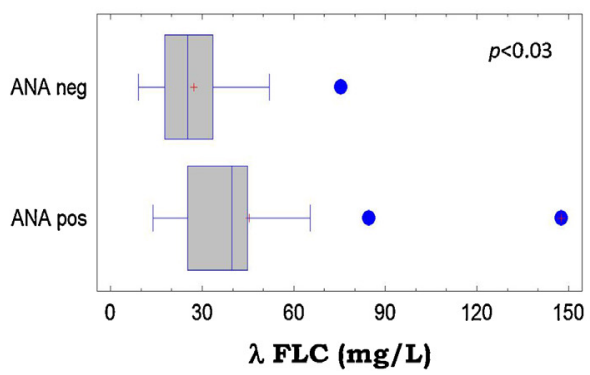

of Internal Medicine, Foundation "A. Gemelli" Hospital. Among them, 50 subjects resulted anti-HCV positive after routine screenings performed before undergoing different clinical procedures. Three/fifty patients had HCV-RNA undetectable in the serum and 8 patients of the remained 47 had no evidences of circulating CGs.

Thirty-nine HCV-RNA and CGs positive patients (mean age $=61.7 \mathrm{yrs}, \pm 1.5,17$ males, $44 \%$ ) were included in the study. Among them, 17 (44\%) resulted positive to ANA test.

The main demographic, clinical and virological characteristics of patients included in the study are outlined in Table 1.

All the patients had normal ALT levels and the Metavir Score, assessed by transient elastography Fibroscan, ranged from F0 to F1.

B Type II and III cryo-patients

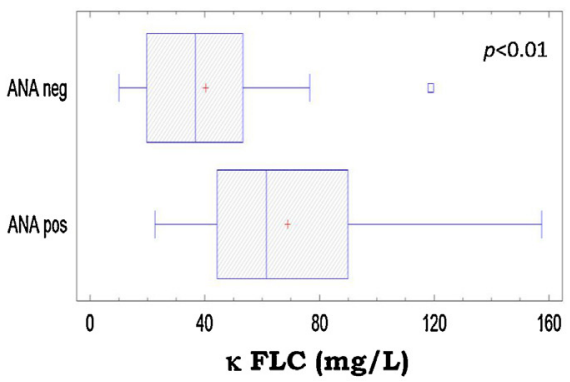

D Type II and III cryo-patients

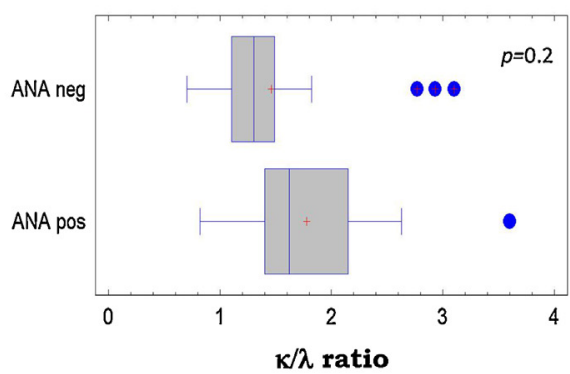

Fig. 1. Rheumatoid factor IgG (RF-IgG), free light chains $\kappa$ and $\lambda$ (FLC $\kappa$, FLC $\lambda$ ) levels and $\kappa / \lambda$ ratio in the study population (type II and III cryo-patients). 
Type II cryo-patients

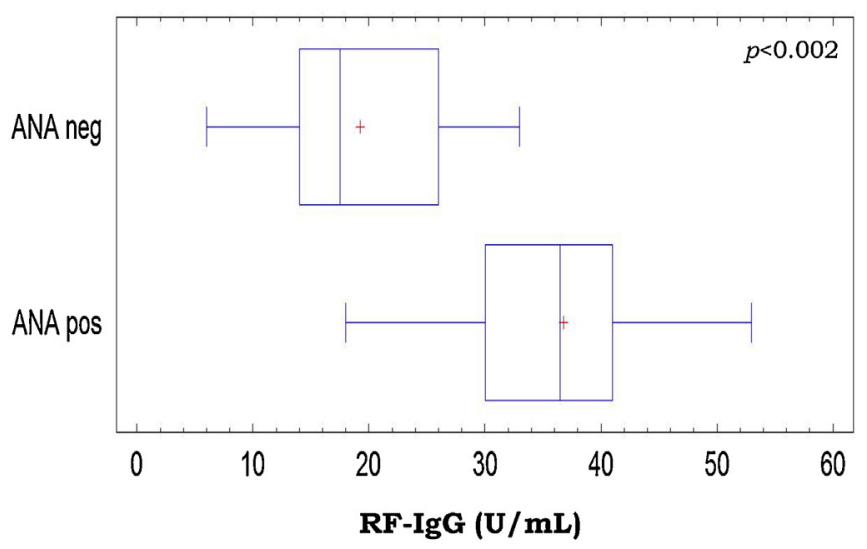

B Type III cryo-patients

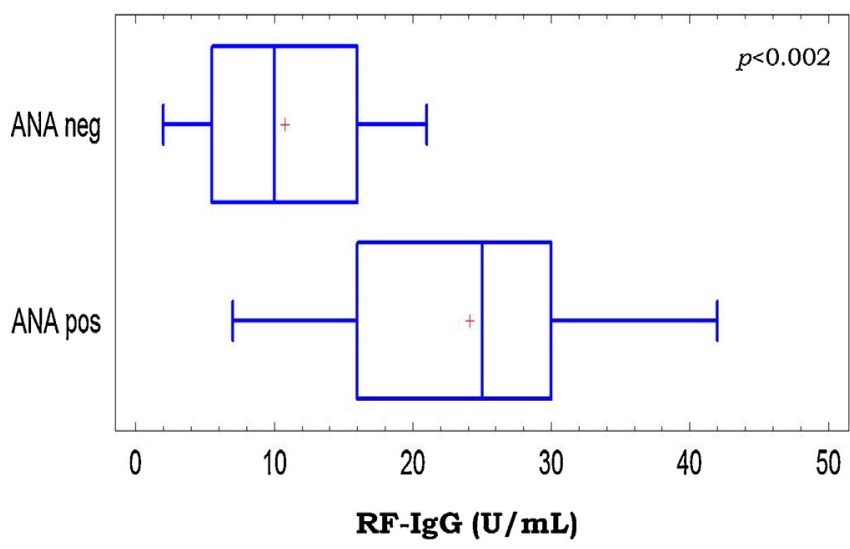

Fig. 2. Rheumatoid factor IgG (RF-IgG) levels in HCV-patients with type II and type II circulating cryoglobulins (CGs).

ANA positive patients showed a higher mean cryocrit and a more frequent alteration of FLC ratio, while no other demographic or clinical differences were observed between the groups.

All samples resulted negative to the test for cryofibrinogenemia and only 6/39 patients showed positive signal in liver-dot, specifically 5 samples resulted positive for LKM1 and 1 sample for SLA (data not shown).

Results obtained from the analyses of RF-IgG, FLC $\kappa$, FLC $\lambda$ levels and the $\kappa / \lambda$ ratio are reported in Fig. 1. A significant difference of RFIgG ranks measured at $37^{\circ} \mathrm{C}$ was observed between ANA negative and ANA positive patients ( $p=0.0002$, panel A), with higher levels shown by the latest ones. Moreover, an increment of both $\kappa$ and $\lambda$ FLC was observed in ANA positive patients respect to the negative ones ( $p<0.01$, panel B; $p<0.03$, panel C, respectively), whereas no significant differences between the two groups were observed concerning the $\kappa / \lambda$ ratio (panel $D$ ).

Also, we evaluated the increment of RF-IgG stratifying for cryoglobulin type. As it is shown in Fig. 2, in both type III and type II cryoglobulin patients, a higher amount of RF-IgG was correlated to ANA positivity.

Moreover, RF-IgG levels resulted different according to cryoglobulinemia type, specifically they appeared lower in type III cryo-patients respect to the type II ones (Fig. 3).

In order to verify the accuracy of the performed tests, we conducted a ROC analysis and we elaborated sensitivity and specificity

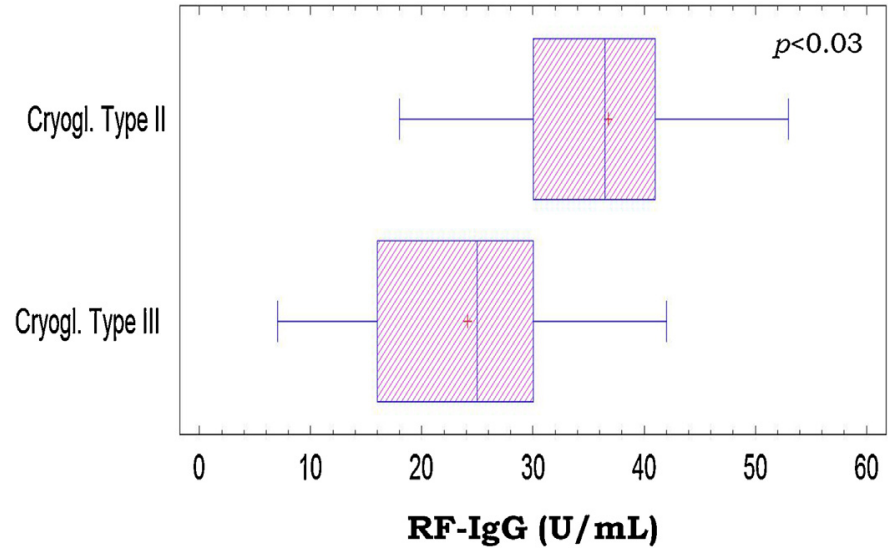

Fig. 3. Rheumatoid factor IgG (RF-IgG) levels stratified according to cryoglobuline type.

ROC curves based on ANA positive vs ANA negative patients. As shown in Fig. 4, the test for RF-IgG has been confirmed to have very high sensitivity (83\%) and specificity (76\%), as well as a remarkable AUC (0.88), while the other tests show lower percentages nevertheless maintaining the statistical significance.

To conclude, we performed a linear discriminant analysis. This analysis allows a reclassification of subjects based on given parameters. We elaborated the algorithm by using FLC kappa, RF-IgG and the $\kappa / \lambda$ ratio since they resulted the most significant parameters from ROC analysis, with the higher AUC. Classification functions coefficients and relative algorithms are shown in Fig. 5.

Using the algorithm we propose, $91 \%$ of positive ANA patients and $76 \%$ of ANA negative patients were correctly reclassified, with the $85 \%$ of total cases properly identify.

\section{Discussion}

HCV related MC is an ideal model to study the complex interactions between autoimmunity and oncogenesis.

Most of the previously published studies analyzed MC symptomatic patients referred to specialized rheumatology/hepatology centres. Conversely, the population we evaluated was composed of subjects without liver disease and with no signs or symptoms of lymphoproliferation and autoimmunity for which the HCV positivity was diagnosed through routine testing, carried out before undergoing a medical procedure. For this reason, the originality and strength of our analysis lies in having tested, for CGs, a population without evidences for suspecting a hidden autoimmunity with accurate sampling and testing.

Surprisingly, we found CGs in the majority of the patients with HCV infection. The percentage appears high, considering the recruitment of an unselected population without any sign/symptoms of autoimmune or lymphoproliferative disease. On the other hand, the Laboratory Department of the Gemelli Hospital that performed all the tests, is renowned for the expertise in detecting the presence of CGs [20].

This epidemiologic finding suggests the opportunity of a more accurate and frequent testing of circulating CGs in HCV-positive patients because it would be beneficial to bring out new cases with a possible risk of evolution into more severe diseases. This earns even greater significance in the new Direct Acting Antivirals era, since the guidelines of all the major associations for the study of the liver indicate the presence of HCV-related MC vasculitis as one of the requirements to be treated, even in the absence of severe liver damage [21].

Although the mono- or oligo-clonal component of CGs with RF activity is usually an IgM, our results showed a high presence of 
A

RF-IgG (U/mL)

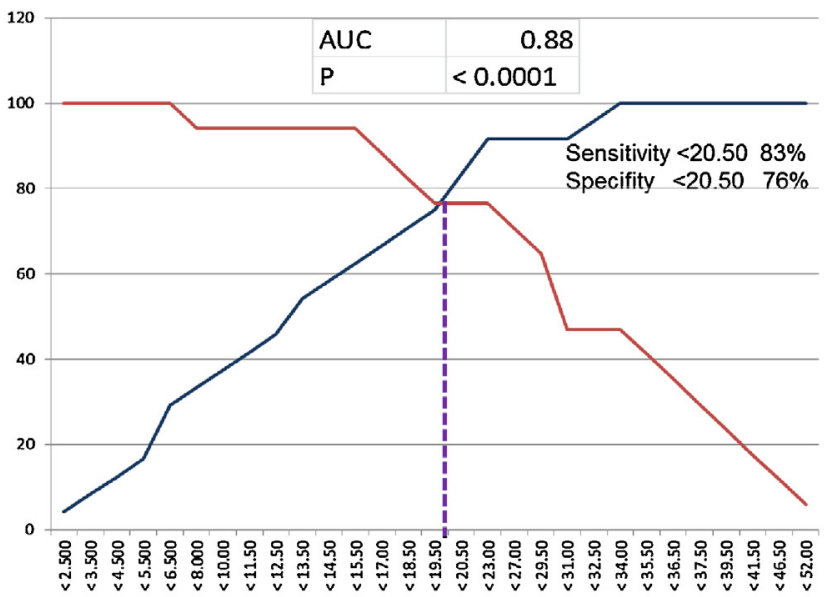

C

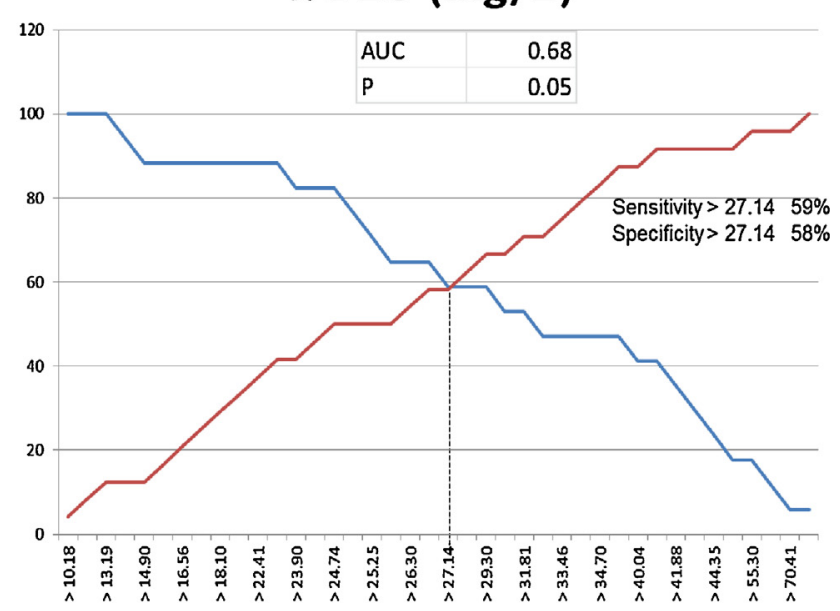

B

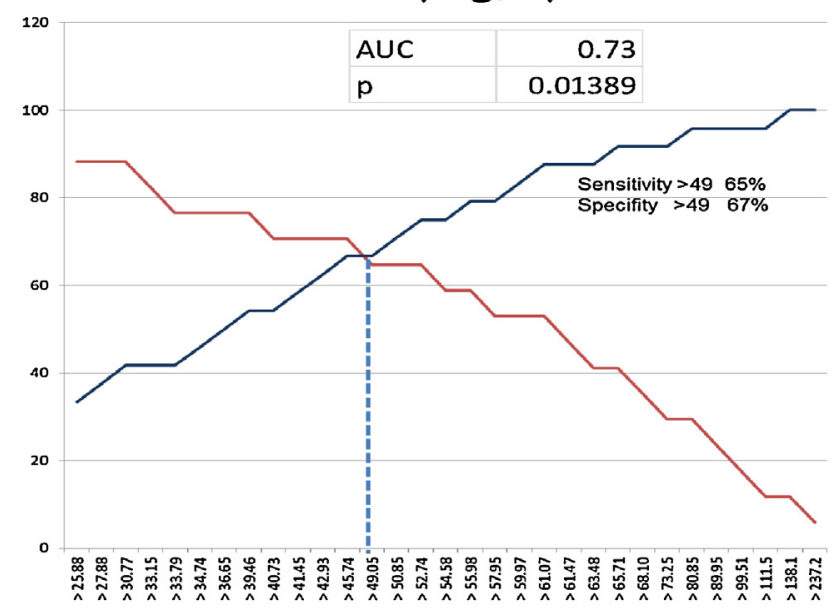

D

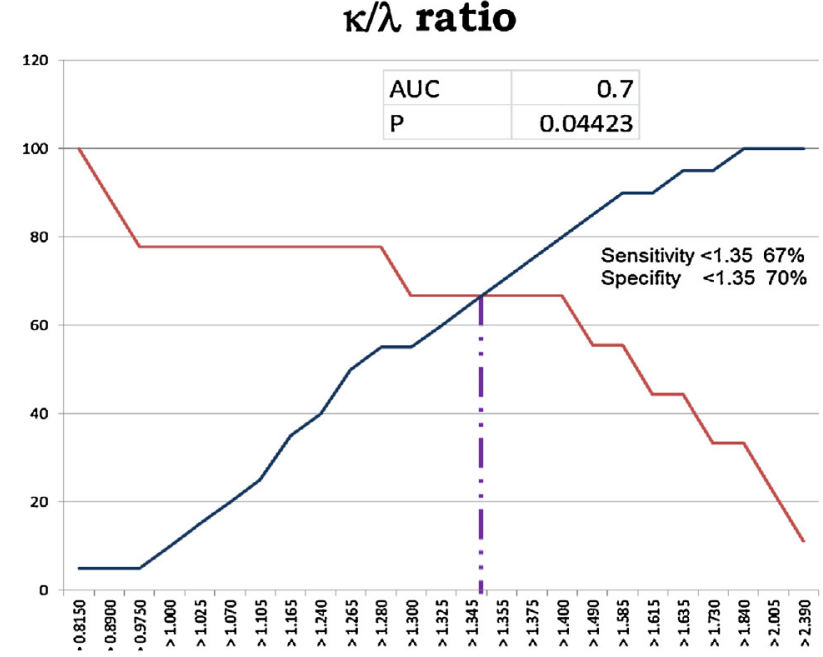

Fig. 4. Sensitivity and specificity ROC curves based on anti-nuclear antibodies (ANA) positive vs ANA negative patients.

RF-IgG in CGs patients. To the best of our knowledge, this is the first study to test the level of RF-IgG in patients with HCV infection and CGs. Despite the interest raised by this finding, we cannot explain its meaning and we are planning to perform further studies in order to understand the role of RF-IgG in HCV-related MC.

FLC serum levels were altered in the majority of HCV-CGs patients and more than $30 \%$ showed an abnormally elevated $\kappa / \lambda$ ratio, completely confirming the value of these serum proteins as markers of HCV-related MC, even in patients without symptoms and with an early-stage disease. More dedicated studies are needed to clarify the pathogenetic role of these molecules: despite FLCs have been considered as "extra-products" of Ig synthesis in the past, it is now recognized that they are very active in many pathways of natural and acquired immunity, interacting with specific immune mediators, surface membrane receptors, and various cells of the immune system [22].

Here, we evaluated the presence of ANA in CGs HCV-patients. The detection of ANA in HCV-infected individuals has been previously described by several authors with percentages lower than $44 \%$ [23-26].

Although conflicting results have been reported on this topic, the majority of authors stated that the ANA positivity is more common in HCV-related advanced liver damage [23-25] and in female gender [24].
Interestingly, symptomatic $\mathrm{MC}$ is a condition more frequently affecting women and that is usually characterized by advanced stages of liver disease.

Our results confirm these previous findings since 62\% of CGpositive and $76 \%$ of ANA-positive patients were females (the difference was not statistically significant).

The determination of circulating CGs has not been performed in these previous works, therefore there is no information available on the ANA-CGs relationship in HCV-infected individuals.

Since our analysis considered only the subgroup of CGs-positive HCV-patients, it is conceivable that we studied a special population enriched in ANA-positive patients: this could explain the higher percentage of ANA positivity we found, compared to previous reports. Also, our data suggest that the production of ANA seems strictly related to CGs production.

Considering the stratification of CGs-HCV-patients in ANApositive and ANA-negative patients, we showed that RF-IgG and FLC levels are significantly higher in the ANA-positive population. Furthermore, the linear discriminant analysis shows the possibility of formulating an algorithm based on three parameters (RF-IgG, FLC $\kappa$ and $\kappa \lambda$ ratio) which can predict ANA positivity/negativity in HCV patients with circulating CGs. This is a clear, further proof that all these different biohumoral parameters are closely correlated and complement each other. 


\begin{tabular}{|cccc|}
\hline Actual ANA & Group size & $\begin{array}{c}\text { Predicted } \\
\text { ANA neg }\end{array}$ & $\begin{array}{c}\text { Predicted } \\
\text { ANA pos }\end{array}$ \\
negative & 22 & $20(91 \%)$ & $2(9 \%)$ \\
positive & 17 & $4(24 \%)$ & $13(76 \%)$ \\
\hline
\end{tabular}

Percentage of cases correctly classified: $85 \%$

\begin{tabular}{|l|c|c|}
\hline \multicolumn{3}{|c|}{ Classification functions coefficients } \\
\hline \hline & negative & positive \\
\hline RF IgG $37^{\circ} \mathrm{C}$ & 0.08 & 0.24 \\
\hline$\kappa / \lambda$ ratio $\mathrm{K} / \mathrm{L}$ & 3.21 & 3.09 \\
\hline$\kappa \mathrm{FLC}$ & -0.005 & -0.001 \\
\hline CONSTANT & -3.53 & -7.34 \\
\hline
\end{tabular}

$\mathrm{Neg}=-3.53(0.08 \mathrm{RF}-\mathrm{IgG}(\mathrm{U} / \mathrm{mL}))+(3.21 \mathrm{\kappa} / \lambda \mathrm{ratio})+(0.005 \mathrm{\kappa LC}(\mathrm{mg} / \mathrm{L}))$

Pos $=-7.34(0.24 \mathrm{RF}-\mathrm{IgG}(\mathrm{U} / \mathrm{mL}))+(3.09 \kappa / \lambda$ ratio $)+(0.001 \kappa \mathrm{FLC}(\mathrm{mg} / \mathrm{L}))$

\section{Linear Discriminant Analysis}

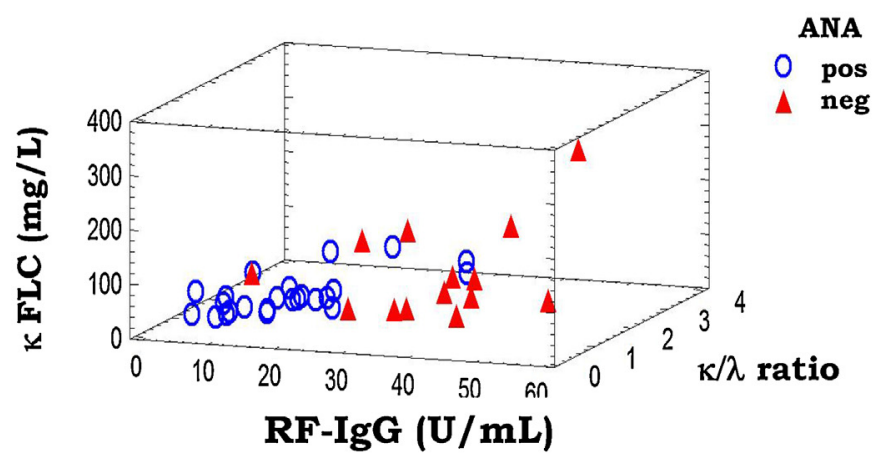

Fig. 5. Linear discriminant analysis and related algorithm.

The immunological tolerance represents the loss of the immune system ability to respond to a particular antigen. The molecular mimicry between viral and self-antigens can cause cross-reaction against self-antigens themselves. HCV-patients show a wide range of autoantibodies usually at very low titres. Among them, ANApositivity was found in $10-33 \%$ of HCV-patients [27]. Although an interpretation of the mechanism responsible for ANA onset and their clinical meaning are not clear, the molecular mimicry could, at least in part, explain this phenomenon. In fact, a sequence homology has been reported between some HCV antigens and nuclear components of host cells. The reactivity against specific viral proteins could also justify the presence of circulating CGs that represent the first step of $\mathrm{B}$-cell clone activation and are the sign of a latent, sub-clinical and not yet symptomatic auto-reactivity.

The persistence of the antigenic stimulus leads to the secretion of IgGs that, in some cases, show RF activity. The biochemical nature of these latter IgGs could be IgG3 subtype [7]. It is conceivable that, after this first phase characterized by asymptomatic IgG RF, the next step would be the appearance of IgM-RF possibly determining a clinically evident disease. In other words, this study open the way for further analyses able to clarify the mechanisms involved.

Indeed, it is conceivable that the more consistent involvement of the immune system, as suggested by the increased levels of FRIgG and FLC observed in CGs-ANA-positive patients, corresponds to a high propensity to evolve towards symptomatic vasculitis.
Prospective dedicated studies based on a long-term follow up of this special population, will hopefully help in ascertain this hypothesis.

In the future, it will be interesting to evaluate these parameters after an effective antiviral treatment. In fact, the patients described in this study did not undergone any antiviral therapy, since, according to the AIFA (Agenzia Italiana del Farmaco; Italian Medicines Agency) current indications, they were not eligible to treatment with DAAs (http://www.agenziafarmaco.gov. it/it/content/aggiornato-1\%E2\%80\%99algoritmo-la-scelta-dellaterapia-1\%E2\%80\%99epatite-c-cronica). In conclusion, our preliminary results outline the importance of RF-IgG and FLCs in asymptomatic CGs and HCV-positive patients, suggesting their use as biomarkers of the evolution of an underlying autoimmune-LPD. Also, the possibility of identifying risk subpopulations may open new scenarios to targeted treatment strategies of HCV patients in extremely early phases (sub-clinical) for diseases characterized by unfavourable progression in an unpredictable time-course.

\section{Conflict of interest}

None declared.

\section{Funding}

LG was supported by a "Fondazione Umberto Veronesi" fellowship; EF was supported by an A.I.R.C. fellowship; the work of the 
Center for Systemic Manifestations of Hepatitis Viruses (MaSVE) of the University of Florence was supported by grants from the "Associazione Italiana per la Ricerca sul Cancro” (AIRC), IG 2015 Id.17391, "Istituto Toscano Tumori" (ITT) and "Ente Cassa di Risparmio di Firenze".

\section{References}

[1] Zignego AL, Giannini C, Gragnani L. HCV and lymphoproliferation. Clinical and Developmental Immunology 2012;2012:980942.

[2] Cacoub P, Gragnani L, Comarmond C, et al. Extrahepatic manifestations of chronic hepatitis C virus infection. Digestive and Liver Disease 2014;46(S5): S165-73.

[3] Zignego AL, Gragnani L, Piluso A, et al. Virus-driven autoimmunity and lymphoproliferation: the example of HCV infection. Expert Review of Clinical Immunology 2015;11:15-31.

[4] Ferri C, Zignego AL, Pileri SA. Cryoglobulins. Journal of Clinical Pathology 2002;55:4-13.

[5] Brouet JC, Clauvel JP, Danon F, et al. Biologic and clinical significance of cryoglobulins. A report of 86 cases. American Journal of Medicine 1974;57:775-88.

[6] Tucci F, Kuppers R. Role of hepatitis C virus in B cell lymphoproliferations. Virologica Sinica 2014;29:3-6.

[7] Basile U, Gulli F, Torti E, et al. Anti-nuclear antibody detection in cryoprecipitates: distinctive patterns in hepatitis $C$ virus-infected patients. Digestive and Liver Disease 2015;47:50-6.

[8] Lenzi M, Bellentani S, Saccoccio G, et al. Prevalence of non-organ-specific autoantibodies and chronic liver disease in the general population: a nested case-control study of the Dionysos cohort see comments. Gut 1999;45: 435-41.

[9] Saadoun D, Elalamy I, Ghillani-Dalbin P, et al. Cryofibrinogenemia: new insights into clinical and pathogenic features. American Journal of Medicine 2009:122:1128-35

[10] Anderson KC, Alsina M, Bensinger W, et al. Multiple myeloma. Journal of the National Comprehensive Cancer Network 2011;9:1146-83.

[11] Dispenzieri A, Kyle R, Merlini G, et al. International Myeloma Working Group guidelines for serum-free light chain analysis in multiple myeloma and related disorders. Leukemia 2009;23:215-24.

[12] Ludwig H, Miguel JS, Dimopoulos MA, et al. International Myeloma Working Group recommendations for global myeloma care. Leukemia 2014:28:981-92.
[13] Rajkumar SV, Kyle RA, Therneau TM, et al. Serum free light chain ratio is an independent risk factor for progression in monoclonal gammopathy of undetermined significance. Blood 2005;106:812-7.

[14] Basile U, Gragnani L, Piluso A, et al. Assessment of free light chains in HCV-positive patients with mixed cryoglobulinaemia vasculitis undergoing rituximab treatment. Liver International 2015.

[15] Kallemuchikkal U, Gorevic PD. Evaluation of cryoglobulins. Archives of Pathology and Laboratory Medicine 1999;123:119-25.

[16] Michaud M, Moulis G, Balardy L, et al. Cryofibrinogenemia: a single-center study at the University Hospital of Toulouse, France. Revue de Medecine Interne 2015;36:237-42.

[17] Meroni PL, Schur PH. ANA screening: an old test with new recommendations. Annals of the Rheumatic Diseases 2010;69:1420-2.

[18] Agmon-Levin N, Damoiseaux J, Kallenberg C, et al. International recommendations for the assessment of autoantibodies to cellular antigens referred to as anti-nuclear antibodies. Annals of the Rheumatic Diseases 2014;73:17-23.

[19] Manns MP, Lohse AW, Vergani D. Autoimmune hepatitis - update 2015. Journal of Hepatology 2015;62:S100-11.

[20] Romitelli F, Pucillo LP, Basile U, et al. Comparison between the traditional a rapid screening test for cryoimmunoglobulins detection. BioMed Research International 2015;2015:783063.

[21] Recommendations for testing, managing, and treating hepatitis C, http://www. hcvguidelines.org; 2014 [accessed 24.04.14].

[22] Redegeld FA, Thio M, Groot Kormelink T. Polyclonal immunoglobulin free light chain and chronic inflammation. Mayo Clinic Proceedings 2012;87:1032-3, author reply 3 .

[23] Lenzi M, Bellentani S, Saccoccio G, et al. Prevalence of non-organ-specific autoantibodies and chronic liver disease in the general population: a nested case-control study of the Dionysos cohort. Gut 1999;45:435-41.

[24] Cassani F, Cataleta M, Valentini P, et al. Serum autoantibodies in chronic hepatitis C: comparison with autoimmune hepatitis and impact on the disease profile. Hepatology 1997;26:561-6.

[25] Gregorio GV, Choudhuri K, Ma Y, et al. Mimicry between the hepatitis C virus polyprotein and antigenic targets of nuclear and smooth muscle antibodies in chronic hepatitis $C$ virus infection. Clinical and Experimental Immunology 2003:133:404-13.

[26] Noda K, Enomoto N, Arai K, et al. Induction of antinuclear antibody after interferon therapy in patients with type-C chronic hepatitis: its relation to the efficacy of therapy. Scandinavian Journal of Gastroenterology 1996:31:716-22.

[27] Lunel F. Hepatitis C virus and autoimmunity: fortuitous association or reality? Gastroenterology 1994;107:1550-5. 